\title{
Multimorbilidade em Portugal: Dados do Primeiro Inquérito Nacional de Saúde com Exame Físico
}

\author{
Multimorbidity in Portugal: Results from The First \\ National Health Examination Survey
}

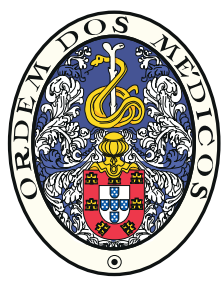

\author{
Guilherme QUINAZ ROMANA®1,2, Irina KISLAYA ${ }^{2,3}$, Mário Rui SALVADOR ${ }^{2,4}$, Susana CUNHA GONÇALVES², \\ Baltazar NUNES 2,3 , Carlos DIAS 2,3 \\ Acta Med Port 2019 Jan;32(1):30-37 • https://doi.org/10.20344/amp.11227
}

\section{RESUMO}

Introdução: A presença de múltiplas doenças crónicas, em simultâneo, no mesmo indivíduo é um problema de saúde reconhecido. Os doentes com multimorbilidade têm necessidades de saúde acrescidas, o que representa um ónus elevado para os cuidados de saúde. Embora não exista uma definição consensual do conceito, a multimorbilidade é definida habitualmente pela presença de duas ou mais doenças crónicas. A existência de evidência, para a realidade nacional, quanto à multimorbilidade poderá contribuir para a gestão e tratamento destes doentes de forma mais eficiente.

Material e Métodos: Com o objetivo de estimar a prevalência de multimorbilidade e identificar os fatores associados foi realizado um estudo epidemiológico transversal com base nos dados do INSEF, um inquérito de base populacional desenvolvido com uma amostra probabilística representativa da população portuguesa $(n=4911)$. A prevalência de multimorbilidade foi estimada para o total da população e para cada um dos sexos, estratificada por grupo etário, região de saúde, educação e rendimento. As magnitudes das associações foram medidas pelas razões de prevalências ajustadas calculadas pelo modelo de regressão de Poisson.

Resultados: A prevalência de multimorbilidade foi de 38,3\% (IC 95\%: 35,4\% a 41,3\%), com maior frequência nas mulheres, nos indivíduos mais velhos, nas regiões de Lisboa e Vale do Tejo, Norte, Algarve e Alentejo e em níveis educacionais mais baixos. Não foi observada associação estatisticamente significativa entre a multimorbilidade e o rendimento.

Discussão: A multimorbilidade é um problema que afeta mais de um terço da população portuguesa. O conhecimento epidemiológico sobre a multimorbilidade em Portugal permite identificar os grupos populacionais onde esta realidade é mais prevalente.

Conclusão: Os valores observados apontam para maior risco de multimorbilidade entre os indivíduos mais velhos e menos diferenciados e está em consonância com os resultados da literatura. Estes dados demonstram a relevância dos doentes com multimorbilidade e têm especial importância na forma com os cuidados de saúde são organizados e prestados.

Palavras-chave: Doenças Crónicas; Inquéritos Epidemiológicos; Multimorbilidade; Portugal

\section{ABSTRACT}

Introduction: The simultaneous presence of multiple chronic diseases in the same individual is recognized as an important public health problem. Patients with multimorbidity have greater healthcare needs, which represents a higher burden on health services. Although there is no consensual definition of this concept, multimorbidity is usually defined as the presence of two or more chronic diseases in the same patient. The existence of evidence regarding multimorbidity will lead to more efficient management and treatment of these patients.

Material and Methods: In order to estimate the prevalence of multimorbidity and to identify the associated factors, a cross-sectional epidemiological study was developed based on data from the INSEF, a population-based survey conducted on a representative probability sample of the Portuguese population $(n=4911)$. The prevalence of multimorbidity was estimated for the total population and separately for men and women, stratified by age group, region, education and income. The magnitudes of the associations were measured by the adjusted prevalence ratios calculated by the Poisson regression model.

Results: Prevalence of multimorbidity was $38.3 \%$ (95\% Cl: $35.4 \%$ to $41.3 \%)$, with higher frequency in women, older people, Lisbon and Tagus Valley; Northern Portugal; Algarve and Alentejo regions and in those with lower academic qualifications. No association was found between multimorbidity and income.

Discussion: Multimorbidity affects more than one third of the Portuguese population. Epidemiological data about multimorbidity in Portugal allows the identification of population groups with higher multimorbidity prevalence.

Conclusion: Our results, which highlight the greater risk of multimorbidity among older and less instructed people, are in line with the literature. These results show the relevance of multimorbidity patients and are especially important in the way how healthcare is organized and provided.

Keywords: Chronic Diseases; Health Surveys; Multimorbidity; Portugal

\section{INTRODUÇÃO}

A multimorbilidade, definida habitualmente na literatura médica pela presença de duas ou mais doenças crónicas no mesmo individuo, ${ }^{1}$ é um problema relevante de saúde pública que importa considerar. ${ }^{2}$
A evolução da medicina e o aumento da esperança de vida reforçaram a pressão que os sistemas de saúde enfrentam com os doentes com múltiplas doenças crónicas. $^{3}$ Os indivíduos com multimorbilidade terão uma maior

1. Unidade de Saúde Pública Lisboa Norte. Agrupamento de Centros de Saúde de Lisboa Norte. Lisboa. Portugal.

2. Departamento de Epidemiologia. Instituto Nacional de Saúde Doutor Ricardo Jorge. Lisboa. Portugal.

3. Centro de Investigação em Saúde Pública (CISP). Escola Nacional de Saúde Pública. Universidade NOVA de Lisboa. Lisboa. Portugal.

4. Unidade de Saúde Pública Dão Lafões. Agrupamento de Centros de Saúde Dão Lafões. Viseu. Portugal.

$\triangle$ Autor correspondente: Guilherme Quinaz Romana. gquinaz.romana@gmail.com

Recebido: 31 de agosto de 2018 - Aceite: 12 de novembro de 2018 | Copyright $\odot$ Ordem dos Médicos 2019 
probabilidade de necessidades de saúde acrescidas e de representarem um maior volume de trabalho dos serviços de saúde. ${ }^{4,5}$ Não obstante os avanços terapêuticos, estes doentes poderão apresentar maior risco de complicações e maior dificuldade de adesão a regimes terapêuticos, por vezes complexos, ${ }^{6}$ com aumento da mortalidade e redução da capacidade funcional. ${ }^{7}$

Na Europa, estima-se que a multimorbilidade represente $70 \%$ a $80 \%$ da despesa em saúde em países como a Dinamarca e seja a causa de oito em cada 11 internamentos hospitalares no Reino Unido. ${ }^{5}$ Os doentes com multimorbilidade são responsáveis por até $78 \%{ }^{4}$ das consultas nos cuidados de saúde primários com uma média de contactos que pode variar entre as $9,35^{4}$ e as $18,6^{6}$ consultas por ano, apresentando um acréscimo entre 5,64 e 12,56 contactos por ano em comparação com indivíduos sem multimorbilidade. Estes doentes revelam também um elevado número de prescrições de medicamentos e de referenciações hospitalares, estimando-se uma média anual de 27,5 prescrições farmacológicas e 0,5 referenciações hospitalares, comparativamente à 15,3 prescrições e 0,3 referenciações nos doentes com apenas uma patologia crónica. ${ }^{6}$

Em 2005/2006 em Portugal, segundo os dados do Inquérito Nacional de Saúde, 36\% da população apresentava três ou mais doenças crónicas sendo o sexo feminino mais afetado $(42,5 \%$ vs $27,4 \%)$ assim como a população com 65 anos ou mais anos - $(55,2 \%$ vs $14,8 \%) .{ }^{8}$ Uma análise mais recente dos dados do SHARE 2011/2012 revelou uma prevalência de multimorbilidade em Portugal, na população com 50 ou mais anos, de $39,4 \% .^{9}$ Um outro estudo, focado na população com 18 ou mais anos de idade que frequentava os cuidados de saúde primários em Portugal continental em 2013/2014, concluiu que $72,7 \%$ dessa população tinha duas ou mais doenças crónicas e verificou uma associação entre níveis educacionais baixos e rendimento económico insuficiente e maior prevalência de multimorbilidade. ${ }^{10} \mathrm{~A}$ nível internacional os estudos apresentam valores variáveis, com prevalências entre $20 \%$ e $40 \% .{ }^{11}$ Os estudos de prevalência revelam, também, a existência de uma relação entre indivíduos desfavorecidos e com baixas habilitações literárias, e a presença de multimorbilidade, , $^{9,12}$ em linha com os resultados nacionais.

Assim, torna-se claro a existência de valores díspares de multimorbilidade nos estudos nacionais já realizados, fruto de desenhos de estudo que utilizam metodologias distintas com populações alvo e grupos etários diferentes. Por conseguinte, é particularmente relevante compreender o padrão atual de multimorbilidade em Portugal, num estudo de base populacional, com um intervalo etário alargado.

É objectivo do presente trabalho descrever e caracterizar a prevalência de multimorbilidade em Portugal no ano de 2015. Pretende-se ainda identificar os fatores associados à multimorbilidade.

\section{MATERIAL E MÉTODOS \\ Fonte de dados}

Este estudo tem como unidade de observação todos os indivíduos que responderam ao Inquérito Nacional de Saúde com Exame Físico (INSEF), desenvolvido pelo Instituto Nacional de Saúde Doutor Ricardo Jorge (INSA) em colaboração com as cinco administrações regionais de saúde do continente, as secretarias regionais de saúde e dos assuntos sociais das regiões autónomas dos Açores e da Madeira e o Instituto Norueguês de Saúde Pública entre 2013 e 2016. O INSEF é um estudo epidemiológico observacional, transversal de prevalência. Este inquérito inclui três componentes distintos: o exame físico; a avaliação analítica e um questionário geral de saúde. Os procedimentos executados foram realizados em conformidade com as recomendações do Inquérito Europeu de Saúde com Exame Físico (EHES). ${ }^{13}$

\section{População alvo e amostra}

A população alvo do INSEF é constituída por indivíduos não-institucionalizados, com idade compreendida entre os 25 e 74 anos residentes em Portugal. A seleção dos participantes foi feita por amostragem probabilística por grupos em duas etapas, estratificada por região de saúde e tipologia de área urbana. ${ }^{13} \mathrm{~A}$ dimensão da amostra foi estabelecida de forma a ser possível estimar uma prevalência esperada de $50 \%$, com uma precisão absoluta de $5 \%$ para um intervalo de confiança a 95\%, em cada região de saúde do continente ou Região Autónoma (RA), considerando um efeito do desenho da amostra de 1,5. O cálculo da dimensão da amostra mínima necessária resultou em 600 indivíduos a nível regional e 4200 a nível nacional, sendo a amostra efectiva final obtida de 4911 indivíduos. ${ }^{13}$

\section{Variáveis em estudo}

As variáveis de interesse para o presente estudo foram colhidas através de um questionário estruturado por entrevista assistida por computador realizada por profissionais de saúde que completaram uma formação para o efeito.

A área de inquirição referente à doença crónica teve como objetivo recolher informações sobre a existência, ou não, de doenças crónicas com mais de seis meses de duração. A variável multimorbilidade foi definida pela presença simultânea de duas ou mais doenças crónicas auto reportadas, de uma lista de vinte patologias (hipertensão arterial; enfarte agudo do miocárdio; acidente vascular cerebral; disritmia cardíaca; diabetes; insuficiência renal crónica; cirrose; hepatite crónica; asma; doença pulmonar obstrutiva crónica; dor crónica; osteoporose; artrite reumatóide; artrose; cancro; depressão; ansiedade crónica; úlcera gástrica ou duodenal; hipercolesterolémia e alergia), cuja seleção se baseou nos anteriores inquéritos nacionais de saúde ${ }^{14,15}$ e no EHES, ${ }^{16}$ assim como no Plano Nacional de Saúde 2013-2016. ${ }^{13,17}$

Foram também selecionadas variáveis de caracterização sociodemográfica, nomeadamente sexo; grupo etário (25 - 34; 35 - 44; 45 - 54; 55 - 64; 65 - 74); educação (Não sabe ler nem escrever /primeiro ciclo; segundo ciclo/ terceiro ciclo; secundário; superior) rendimento mensal por adulto equivalente (segundo escala da OCDE) e região de 
saúde.

\section{Análise estatística}

Para descrever as variáveis em estudo, foram calculadas frequências absolutas e relativas.

A prevalência de multimorbilidade e os respetivos intervalos de confiança a $95 \%$ foram estimados para o total da população e para cada um dos sexos, estratificados por grupo etário, região de saúde, educação e rendimento.

Para identificar os fatores associados à multimorbilidade foram calculadas as razões de prevalências brutas (RP), e os respetivos intervalos de confiança a $95 \%$.

Para além de se testar a associação entre a multimorbilidade e as variáveis independentes, duas a duas, procedeu-se a uma abordagem multivariada. Os modelos de regressão de Poisson, que permitiram obter as razões de prevalência ajustadas (aRP), foram construídos separadamente para cada um dos sexos e ajustados para as restantes variáveis independentes. O nível de significância foi estabelecido em $5 \%$.

A análise estatística foi desenvolvida com base nos pesos amostrais. ${ }^{13}$ Foram utilizados os softwares IBM SPSS Statistics (versão 25$)^{18}$ no módulo complex samples e Stata (versão 15.1$)^{19}$ no módulo survey data analysis (SVY).

\section{Questões éticas}

O estudo analisou dados provenientes do INSEF sem recolha de nova informação. O INSEF foi aprovado pela Comissão Nacional de Proteção de Dados (Autorização $\left.n^{\circ} 9348 / 2010\right)$, assim como pelas comissões de ética do Instituto Nacional de Saúde Doutor Ricardo Jorge; das diferentes administrações regionais de saúde e dos hospitais da Horta e Centro Hospitalar de Lisboa Ocidental. Foi obtido um consentimento informado de todos os participantes. Não obstante, o presente estudo foi também aprovado pela Comissão de Ética do Instituto Nacional de Saúde Doutor Ricardo Jorge

\section{RESULTADOS}

Dos 4911 indivíduos que participaram no INSEF, 52,5\% foram do sexo feminino. A faixa etária mais representada foi a dos 35 - 44 anos com 23,5\%, seguida dos escalões etários 45 - 54 (22,4\%) e 55 - 64 (19,9\%). Cerca de um terço dos participantes tinha como habilitações literárias o segundo e terceiros ciclos $(31,5 \%)$, seguindo-se os indivíduos que não sabem ler nem escrever, ou têm apenas o primeiro ciclo $(27,7 \%)$. Quanto ao rendimento, as classes média-alta e alta tinham a maior representatividade - 41,5\%. As classes media-baixa e baixa surgiam com $38,2 \%$ e a classe média com $20,4 \%$. A maioria dos indivíduos reside na região Norte do país $(35,4 \%)$. As segundas e terceiras regiões mais representadas foram, respetivamente, Lisboa e Vale do Tejo e o Centro. Do total de participantes, 42,2\% referiram não ter nenhuma doença crónica; $19,4 \%$ referiram ter uma doença crónica; $17,0 \%$ duas doenças crónicas e $10,4 \%$ três doenças crónicas (Tabela 1).

Para ambos os sexos, as duas patologias mais preva- lentes foram a hipertensão, $25,1 \%$ nos homens e $26,1 \%$ nas mulheres, e a hipercolesterolémia, respetivamente $23,7 \%$ e $25,7 \%$. No sexo feminino a terceira patologia mais frequentemente reportada foi a artrose $(20,6 \%)$, e nos homens foram as alergias $(11,4 \%)$ - (Apêndice 1: https://www. actamedicaportuguesa.com/revista/index.php/amp/article/ view/11227/Apendice_01.pdf).

A prevalência de multimorbilidade na população foi de $38,3 \%$ IC 95\% (35,4 a 41,3\%). Verificou-se uma maior prevalência de multimorbilidade no sexo feminino $(43,4 \%$ vs $32,7 \%, \mathrm{RP}=1,33$ ) (Tabela 2).

Os valores de prevalência foram semelhantes entre homens e mulheres nas faixas etárias dos 25 - 34 (9,5\% vs $9,4 \%)$ e 35 - 44 anos (15,2\% vs 18,0\%). Nos escalões etários seguintes as mulheres apresentaram uma prevalência superior comparativamente aos homens. Nas mulheres o valor máximo encontrado foi de $82,3 \%$, na faixa etária 65 74 anos, e de $65,5 \%$ nos homens para a mesma idade.

Observou-se um aumento estatisticamente significativo da prevalência de multimorbilidade com a idade, para ambos os sexos. Nas mulheres, comparativamente à faixa etária dos 25 - 34 anos, a prevalência quase duplicou para a faixa etária dos $35-44$ anos $(R P=1,90)$. Nos escalões etários seguintes a prevalência de multimorbilidade foi de 4,58 (45 - 54 anos), 7,54 (55 - 64 anos) e 8,71 (65 - 74 anos) vezes superior, respetivamente, em comparação com as mais jovens. Nos homens, as diferenças estatisticamente significativas na prevalência de multimorbilidade verificaram-se somente entre os indivíduos com 25 - 34 anos e os indivíduos com 55 - 74 anos de idade, sendo as respetivas razões de prevalência 6,39 para faixa etária 5564 anos e 6,92 para faixa etária 65 - 74 anos (Tabela 2).

Os grupos populacionais com maiores habilitações literárias apresentaram uma menor prevalência de multimorbilidade para ambos os sexos. Para as mulheres, verificou-se uma diminuição gradual da prevalência de multimorbilidade com aumento de nível de escolaridade, de 70,0\% no grupo sem escolaridade ou com o primeiro ciclo do ensino básico, para os $25 \%$ no grupo com habilitações ao nível do ensino superior. Para os homens a prevalência de multimorbilidade variou segundo o nível de escolaridade entre $57,8 \%$ e $19,7 \%$. Em comparação com o grupo sem escolaridade ou com o primeiro ciclo do ensino básico, a prevalência diminui em $53 \%(R P=0,47)$ para o grupo de segundo ciclo / terceiro ciclo, em $66 \%(R P=0,34)$ para o grupo de ensino secundário e em $63 \%(R P=0,37)$ para o grupo do ensino superior.

No que se refere às diferentes regiões de saúde foi possível encontrar diferenças estatisticamente significativas na distribuição da prevalência de multimorbilidade para ambos os sexos. Os residentes na RA da Madeira apresentaram uma prevalência mais baixa, $21,3 \%$ e $32,6 \%$ para os homens e mulheres, respetivamente. Tendo esta região como referência, para as mulheres, a prevalência foi superior na RA dos Açores (RP = 1,27), Norte (RP = 1,36), Lisboa e Vale do Tejo $(R P=1,41)$, Algarve $(R P=1,46)$ e Alentejo $(R P=1,50)$. Para os homens observou-se um padrão 
Tabela 1 - Descrição da amostra

\begin{tabular}{|c|c|c|}
\hline & & Freq* $^{*}(n)$ \\
\hline \multirow[t]{2}{*}{ Sexo } & Masculino & $47,5 \%(2646)$ \\
\hline & Feminino & $52,5 \%(2245)$ \\
\hline \multirow{5}{*}{ Idade } & $25-34$ & $18,3 \%(714)$ \\
\hline & $35-44$ & $23,5 \%(1135)$ \\
\hline & $45-54$ & $22,4 \%(1193)$ \\
\hline & $55-64$ & $19.9 \%(1098)$ \\
\hline & $65-74$ & $15,9 \%(771)$ \\
\hline \multirow[t]{7}{*}{ Região } & Norte & $35,4 \%(777)$ \\
\hline & Centro & $16,2 \%(706)$ \\
\hline & Lisboa e Vale do Tejo & $34,8 \%(650)$ \\
\hline & Alentejo & $4,6 \%(690)$ \\
\hline & Algarve & $4,2 \%(644)$ \\
\hline & RA Madeira & $2,5 \%(695)$ \\
\hline & RA Açores & $2,3 \%(749)$ \\
\hline \multirow[t]{4}{*}{ Educação } & Não sabe ler nem escrever $/ 1^{\circ}$ ciclo & $27,7 \%(1516)$ \\
\hline & $2^{\circ} \mathrm{ciclo} / 3^{\circ} \mathrm{ciclo}$ & $31,5 \%(1595)$ \\
\hline & Secundário & $21,4 \%(958)$ \\
\hline & Superior & $19,4 \%(838)$ \\
\hline \multirow[t]{3}{*}{ Rendimento } & Baixo, Médio - baixo & $38,2 \%(2021)$ \\
\hline & Médio & $20,4 \%(872)$ \\
\hline & Médio-alto, Alto & $41,5 \%(1751)$ \\
\hline \multirow[t]{10}{*}{ Doenças Crónicas } & 0 doenças crónicas & $42,2 \%(2055)$ \\
\hline & 1 doença crónica & $19,4 \%(930)$ \\
\hline & 2 doenças crónicas & $17,0 \%(838)$ \\
\hline & 3 doenças crónicas & $10,4 \%(474)$ \\
\hline & 4 doenças crónicas & $5,2 \%(264)$ \\
\hline & 5 doenças crónicas & $3,0 \%(139)$ \\
\hline & 6 doenças crónicas & $1,3 \%(68)$ \\
\hline & 7 doenças crónicas & $0,7 \%(46)$ \\
\hline & 8 doenças crónicas & $0,4 \%(21)$ \\
\hline & 9 doenças crónicas & $0,3 \%(10)$ \\
\hline
\end{tabular}

* Frequências relativas ponderadas para a distribuição da população portuguesa por região de saúde, sexo e grupo etário em 2015

Freq: frequência

semelhante, com exceção da RA Açores.

Não se verificou associação estatisticamente significativa entre a multimorbilidade e o rendimento.

No modelo de regressão multivariável continuou a verificar-se uma relação entre a multimorbilidade e a idade, com uma aRP nos escalões etários 55 - 64 e 65 - 74 de 5,67 (IC 95\%: 2,54 a 12,65) e 5,68 (IC 95\%: 2,47 a 13,05) nos homens e 6,71 (IC 95\%: 4,01 a 11,21) e 7,51 (IC 95\%: 4,52 a 12,51) nas mulheres, em comparação com o escalão etário 25 - 34 (Tabela 3).

A análise multivariável apresentou resultados estatisticamente significativos quanto à distribuição da prevalência de multimorbilidade nas diferentes regiões de saúde. As regiões com uma prevalência mais elevada são, para ambos os sexos, o Alentejo; Lisboa e Vale do Tejo e Algarve.

Quanto à variável educação, os valores observados só foram estatisticamente significativos para o ensino secundário no sexo masculino e para o ensino superior no sexo feminino. Confirmou-se, no entanto, no resultado destes dois grupos, um menor risco de multimorbilidade nos indivíduos com maiores habilitações literárias face aos indivíduos que não sabem ler nem escrever ou têm apenas o primeiro ciclo.

\section{DISCUSSÃO}

O presente estudo focou-se na prevalência de multimorbilidade na população portuguesa, bem como na sua distribuição por sexo, idade, região educação e rendimento. Verificou-se que a multimorbilidade é um problema comum em Portugal, com uma prevalência de $38,3 \%$, especialmente nos indivíduos mais velhos $-65,5 \%$ nos homens e $82,3 \%$ nas mulheres - e com menor diferenciação - $57,8 \%$ 


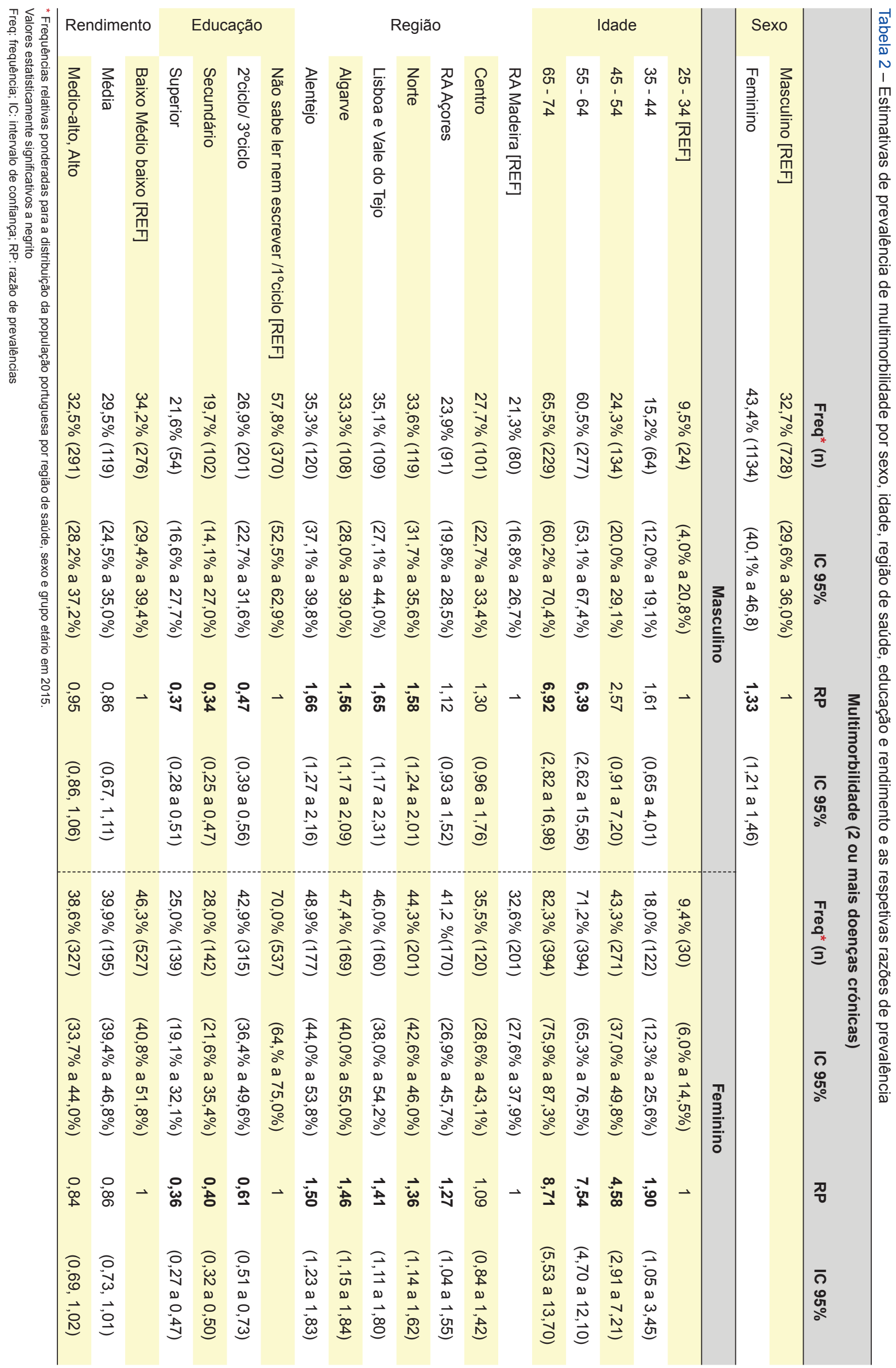


nos homens e 70,0\% nas mulheres.

Os valores de multimorbilidade descritos para a população portuguesa mostram a dimensão desta questão em Portugal, ${ }^{8-10}$ tendo os resultados encontrados reforçado a extensão do problema.

No panorama europeu, o trabalho de Puth et al, na Alemanha, também com base num inquérito populacional em indivíduos com 18 ou mais anos de idade, revela uma prevalência de multimorbilidade de $39,6 \%$, com um aumento da frequência com a idade $-49,2 \%$ dos adultos entre os 50 - 59 anos apresentavam-se com multimorbilidade - e em indivíduos com menores habilitações literárias. ${ }^{20}$ Dados da população espanhola, num trabalho cuja fonte de dados foi o inquérito populacional COURAGE, revelaram um valor de multimorbilidade inferior ao encontrado no presente estudo: 20,0\%. ${ }^{21}$ Também Barnett et al concluiu utilizando os dados dos registos clínicos que a prevalência de multimorbilidade na Escócia é de 23,3\%. ${ }^{7}$

No entanto, as diferenças metodológicas e a inexistência de uma definição consensualmente aceite para a multimorbilidade limita a comparação dos nossos resultados com outros trabalhos. ${ }^{11} \mathrm{O}$ recurso a diferentes medições para o mesmo problema, com a utilização de amostras populacionais distintas e diferente número e tipo de comorbilidades, dificulta a extrapolação dos resultados e dá origem a valores amplos de prevalência. ${ }^{22}$ Dados nacionais apon- tam para a discrepância de resultados quando utilizadas diferentes listas de patologias. Numa mesma população, a definição de multimorbilidade adotada e as doenças crónicas incluídas, influenciam os resultados obtidos. ${ }^{23}$ Também a fonte de dados é variável, embora existam diversos estudos baseados em inquéritos populacionais, ${ }^{20,21,24}$ muitos dos trabalhos nesta área têm como base registos clínicos. ${ }^{4,6,7,10}$

Vários estudos mostram ainda a associação entre multimorbilidade e fatores socioeconómicos ${ }^{25}$ : Os indivíduos que residem em áreas desfavorecidas apresentam-se com multimorbilidade 10 a 15 anos antes, quando comparados com os que habitam noutras zonas. ${ }^{7}$ Os resultados por nós obtidos estão em consonância com os estudos supracitados ao mostrarem uma prevalência superior de multimorbilidade nos indivíduos mais velhos e com habilitações literárias mais baixas. Níveis educacionais mais altos associam-se a um melhor estado de saúde, consequência de comportamentos mais saudáveis e maior literacia, ${ }^{26}$ o que pode justificar uma prevalência inferior de multimorbilidade.

Se tivermos em consideração a variável idade, a prevalência de multimorbilidade encontrada na população mais idosa é superior. A associação entre a maior prevalência de multimorbilidade e a idade é conhecida. Tal deve-se, aparentemente, ao aumento do número de anos vividos com a provável acumulação de doenças crónicas. ${ }^{27,28}$ Dados de

Tabela 3 - Modelo multivariável: Estimativa das razões de prevalência ajustadas de multimorbilidade segundo o grupo etário, região de saúde, educação e rendimento

\begin{tabular}{|c|c|c|c|c|c|}
\hline & & \multicolumn{4}{|c|}{ Multimorbilidade (2 ou mais doenças crónicas) } \\
\hline & & \multicolumn{2}{|c|}{ Masculino } & \multicolumn{2}{|c|}{ Feminino } \\
\hline & & aRP & IC $95 \%$ & aRP & IC $95 \%$ \\
\hline \multirow{5}{*}{ Idade } & 25 - 34 [REF] & 1 & & 1 & \\
\hline & $35-44$ & 1,63 & $(0,72$ a 3,69$)$ & 1,78 & $(0,96$ a 3,32$)$ \\
\hline & $45-54$ & 2,27 & $(0,90$ a 5,72$)$ & 4,17 & $(2,61$ a 6,67$)$ \\
\hline & $55-64$ & 5,67 & $(2,54$ a 12,65$)$ & 6,71 & $(4,01$ a 11,21$)$ \\
\hline & $65-74$ & 5,68 & $(2,47$ a 13,05$)$ & 7,51 & $(4,52$ a 12,51$)$ \\
\hline \multirow[t]{7}{*}{ Região } & RA Madeira [REF] & 1 & & 1 & \\
\hline & Centro & 1,15 & $(0,89$ a 1,47$)$ & 0,99 & $(0,79$ a 1,27$)$ \\
\hline & RAAçores & 1,16 & $(0,91$ a 1,48$)$ & 1,33 & $(1,06$ a 1,67$)$ \\
\hline & Norte & 1,45 & $(1,23$ a 1,71$)$ & 1,31 & $(1,12$ a 1.54$)$ \\
\hline & Lisboa e Vale do Tejo & 1,59 & $(1,19$ a 2,12$)$ & 1,39 & $(1,14$ a 1.69$)$ \\
\hline & Algarve & 1,50 & $(1,23$ a 1,84$)$ & 1,39 & (1.15 a 1.69$)$ \\
\hline & Alentejo & 1,49 & $(1,17$ a 1,90$)$ & 1,41 & (1.17 a 1.71) \\
\hline \multirow[t]{4}{*}{ Educação } & $\begin{array}{l}\text { Não sabe ler nem escrever } \\
/ 1^{\circ} \mathrm{ciclo}[\mathrm{REF}]\end{array}$ & 1 & & 1 & \\
\hline & $2^{\circ} \mathrm{ciclo} / 3^{\circ} \mathrm{ciclo}$ & 0,85 & $(0,64$ a 1,11$)$ & 0,88 & $(0,77$ a 1,02$)$ \\
\hline & Secundário & 0,63 & $(0,47$ a 0,84$)$ & 0,85 & $(0,69$ a 1,05$)$ \\
\hline & Superior & 0,80 & $(0,54$ a 1,18$)$ & 0,75 & $(0,58$ a 0,96$)$ \\
\hline \multirow{3}{*}{ Rendimento } & Baixo, médio baixo [REF] & 1 & & 1 & \\
\hline & Média & 0,94 & $(0,81$ a 1,09$)$ & 0,92 & $(0,81$ a 1,05$)$ \\
\hline & Alto, médio alto & 1,04 & $(0,90$ a 1,19$)$ & 0,99 & $(0,87$ a 1,12$)$ \\
\hline
\end{tabular}

IC: intervalo de confiança; aRP: razão de prevalências ajustada

Valores estatisticamente significativos a negrito. 
16 países europeus mostram que a média de prevalência de multimorbilidade é de $22,7 \%$, na faixa etária entre os 50 e os 59 anos de idade, passando para $52,8 \%$ nos indivíduos com 70 ou mais anos. ${ }^{9}$ Em Portugal, os resultados recentemente publicados de uma coorte focada na população mais idosa, mostram que a prevalência de multimorbilidade nestes indivíduos é de $78,3 \%,{ }^{29}$ valor este semelhante aos resultados encontrados no presente estudo.

Quanto ao sexo, a maior prevalência obtida no sexo feminino é coincidente com os dados da literatura. ${ }^{30}$ As mulheres têm uma esperança média de vida mais elevada, que aliada a fatores biológicos específicos, contribuem para um maior número de doenças crónicas concomitantes, tais como patologias músculo-esqueléticas ou de saúde mental. ${ }^{31}$ Uma outra explicação prende-se com uma maior vigilância para as questões da saúde, o que leva a um maior auto reporte. ${ }^{32}$

As diferenças encontradas na prevalência de multimorbilidade entre as diversas regiões de saúde carecem de maior investigação. Tendo sido realizado o ajustamento para a idade, educação e rendimento, uma posterior investigação poderá considerar, também, diferentes condições de acesso aos serviços de saúde. ${ }^{33}$

Tratando-se de um estudo baseado num inquérito populacional, existe a possibilidade dos 'participantes' serem distintos dos 'não participantes'. Para limitar esta hipótese foi realizado no âmbito do INSEF um inquérito aos indivíduos 'não participantes', sendo assim possível a análise de eventuais desvios entre os dois grupos. De forma a diminuir a possibilidade de diferenças entre a amostra e a população alvo, foram utilizados ponderadores para a idade, sexo e região. ${ }^{13}$

Outras limitações são passíveis de ser mencionadas. O modo de colheita de dados, realizado em centros de saúde, poderá ter deixado de fora os utentes que, preferencialmente, utilizam os serviços de saúde privados: $60 \%$ das consultas de especialidade em Portugal ocorrem no sector privado ou social, $26 \%$ da população portuguesa é subscritora de seguros de saúde voluntários ${ }^{34}$ e $27 \%$ dos medicamentos dispensados nas farmácias têm origem em prescrições da medicina privada. ${ }^{35} \mathrm{~A}$ exclusão dos indivíduos com mais de 75 anos e aqueles que habitam em instituições, pode subestimar a prevalência de multimorbilidade.

Embora a pergunta utilizada no INSEF salvaguarde se o diagnóstico foi feito por um médico, os dados são obtidos por auto reporte. Os inquiridos respondem, também, com informação do passado, tal poderá pressupor a existência de um viés de memória.

A relevância deste estudo prende-se com a necessidade de conhecer a realidade da multimorbilidade no contexto nacional. A sua principal vantagem reside na dimensão da amostra e na representatividade da população portuguesa.

\section{CONCLUSÃO}

A multimorbilidade apresenta-se em Portugal como um problema de magnitude assinalável, atingindo $38,3 \%$ da população com 25 - 74 anos.

De acordo com os resultados encontrados, o risco de multimorbilidade é superior no sexo feminino, nos escalões etários mais velhos e nos indivíduos menos diferenciados.

A existência de um estudo de base populacional, com representatividade nacional, possibilita a melhor caraterização destes doentes em Portugal. Esta evidência permitirá contribuir para a atual discussão quanto à necessidade dos sistemas de saúde se adaptarem aos doentes com múltiplas doenças crónicas, com políticas que permitam tratar melhor e com maior eficiência.

\section{PROTEÇÃO DE PESSOAS E ANIMAIS}

Os autores declaram que os procedimentos seguidos estavam de acordo com os regulamentos estabelecidos pelos responsáveis da Comissão de Investigação Clínica e Ética e de acordo com a Declaração de Helsínquia da Associação Médica Mundial.

\section{CONFIDENCIALIDADE DOS DADOS}

Os autores declaram ter seguido os protocolos do seu centro de trabalho acerca da publicação de dados.

\section{CONFLITOS DE INTERESSE}

Os autores declaram não ter conflitos de interesse.

\section{FONTES DE FINANCIAMENTO}

O $1^{\circ}$ Inquérito Nacional de Saúde com Exame Físico (INSEF 2015), parte integrante do projeto pré-definido do Programa Iniciativas em Saúde Pública "Improvement of epidemiological health information to support public health decision and management in Portugal. Towards reduced inequalities, improved health, and bilateral cooperation", beneficia de um apoio financeiro de cerca de 1.500.000€ concedido pelo Mecanismo Financeiro do Espaço Económico Europeu 2009-2014 através das EEA Grants e pelo Governo de Portugal.

\section{REFERÊNCIAS}

1. Mercer S, Salisbury C, Fortin M. ABC of multimorbidity. London: BMJ Books; 2014.

2. Vogeli C, Shields AE, Lee TA, Gibson TB, Marder WD, Weiss KB, et al. Multiple chronic conditions: prevalence, health consequences, and implications for quality, care management, and costs. J Gen Intern Med. 2007;22:S391-5.

3. Rijken M, Struckmann V, van der Heide I, Hujala A, Barbabella F, van Ginneken E, et al. How to improve care for people with multimorbidity in Europe? Observatory on Health Systems and Policies. ICARE4EU project; 2017
4. Salisbury C, Johnson L, Purdy S, Valderas JM, Montgomery AA. Epidemiology and impact of multimorbidity in primary care: a retrospective cohort study. Br J Gen Pract. 2011;61:12-21.

5. Glynn LG, Valderas JM, Healy P, Burke E, Newell J, Gillespie P, et al. The prevalence of multimorbidity in primary care and its effect on health care utilization and cost. Fam Pract. 2011;28:516-23.

6. van Oostrom SH, Picavet HS, de Bruin SR, Stirbu I, Korevaar JC, Schellevis FG, et al. Multimorbidity of chronic diseases and health care utilization in general practice. BMC Fam Pract. 2014;15:61.

7. Barnett K, Mercer SW, Norbury M, Watt G, Wyke S, Guthrie B. 
Epidemiology of multimorbidity and implications for health care, research, and medical education: a cross-sectional study. Lancet. 2012;380:37-43.

8. Nicolau V, Nunes C, Escoval A. Estudo: prevalência da multimorbilidade em Portugal. In: Congresso Nacional de Saúde Pública. Lisboa: DGS; 2014.

9. Palladino R, Lee JT, Ashworth M, Triassi M, Millett C. Associations between multimorbidity, healthcare utilisation and health status: evidence from 16 European countries. Age Ageing. 2016;45:431-5.

10. Prazeres F, Santiago L. Prevalence of multimorbidity in the adult population attending primary care in Portugal: a cross-sectional study. BMJ Open. 2015;5:e009287.

11. Lefèvre T, d'Ivernois JF, De Andrade V, Crozet C, Lombrail P, Gagnayre R. What do we mean by multimorbidity? An analysis of the literature on multimorbidity measures, associated factors, and impact on health services organization. Rev Epidemiol Sante Publique. 2014;62:305-14.

12. Wang HH, Wang JJ, Lawson KD, Wong SY, Wong MC, Li FJ, et al. Relationships of multimorbidity and income with hospital admissions in 3 health care systems. Ann Fam Med. 2015;13:164-7.

13. Santos AJ, Gil AP, Kislaya I, Antunes L, Barreto M, Namorado S, et al. $1^{\circ}$ Inquérito Nacional de Saúde com Exame Físico (INSEF 2015): relatório metodológico. Lisboa: Instituto Nacional de Saúde Doutor Ricardo Jorge; 2016.

14. Instituto Nacional de Estatística, Instituto Nacional de Saúde Doutor Ricardo Jorge. Inquérito Nacional de Saúde 2005/2006. Lisboa: INE; 2009.

15. Instituto Nacional de Estatística. Inquérito Nacional de Saúde 2014. Lisboa: INE; 2016.

16. Tolonen $\mathrm{H}$, editor. EHES Manual. Part A. Planning and preparation of the survey. $2^{\text {nd }}$ ed. Helsinki: National Institute for Health and Welfare; 2016.

17. Direção Geral da Saúde. Plano Nacional de Saúde 2012-2016. Lisboa: DGS; 2012.

18. IBM Corp. IBM SPSS Statistics for Windows, Version 25.0. Armonk: IBM Corp; 2017.

19. StataCorp. Stata Statistical Software: Release 15. College Station: StataCorp LP; 2017.

20. Puth MT, Weckbecker K, Schmid M, Münster E. Prevalence of multimorbidity in Germany: Impact of age and educational level in a cross-sectional study on 19,294 adults. BMC Public Health. 2017;17:17.
21. Garin N, Olaya B, Perales J, Moneta MV, Miret M, Ayuso-Mateos JL, et al. Multimorbidity patterns in a national representative sample of the Spanish adult population. PLoS One. 2014;9.

22. Violan C, Foguet-Boreu Q, Flores-Mateo G, Salisbury C, Blom J, Freitag $\mathrm{M}$, et al. Prevalence, determinants and patterns of multimorbidity in primary care: a systematic review of observational studies. PLoS One. 2014:9:3-11.

23. Prazeres F, Santiago L. Measuring multimorbidity in family practice - a comparison of two methods. Fam Pract. 2018;1-5.

24. Hoffman C, Rice D, Sung HY. Persons with chronic conditions. Their prevalence and costs. JAMA. 1996;276:1473-9.

25. Pathirana TI, Jackson CA. Socioeconomic status and multimorbidity: a systematic review and meta-analysis. Aust N Z J Public Health. 2018;42:186-94.

26. 2Cohen AK, Syme SL. Education: a missed opportunity for public health intervention. Am J Public Health. 2013;103:997-1001.

27. Fortin M, Bravo G, Hudon C, Vanasse A, Lapointe L. Prevalence of multimorbidity among adults seen in family practice. Ann Fam Med 2005;3:223-8.

28. Marengoni A, Angleman S, Melis R, Mangialasche F, Karp A, Garmen A, et al. Aging with multimorbidity: a systematic review of the literature. Ageing Res Rev. 2011;10:430-9.

29. Rodrigues AM, Gregório MJ, Sousa RD, Dias SS, Santos MJ, Mendes $\mathrm{JM}$, et al. Challenges of ageing in portugal: data from the EpiDoC cohort. Acta Med Port. 2018;31:80-93.

30. Uijen A, van de Lisdonk E. Multimorbidity in primary care: prevalence and trend over the last 20 years. Eur J Gen Pract. 2008;14:S28-32.

31. Vlassoff C. Gender differences in determinants and consequences of health and illness. J Heal Popul Nutr. 2007;25:47-61.

32. Pache B, Vollenweider P, Waeber G, Marques-Vidal P. Prevalence of measured and reported multimorbidity in a representative sample of the Swiss population Disease epidemiology - Chronic. BMC Public Health 2015;15:1-8

33. Observatório Português dos Sistemas de Saúde - OPSS. Viver em tempos incertos: sustentabilidade e equidade na saúde. Relatório de Primavera 2017. Lisboa: OPSS; 2017. p. 1-186.

34. Simões JD, Augusto GF, Fronteira I, Hernández-Quevedo C. Health systems in transition. Portugal Health System Rev. 2017;19.

35. Observatório Português dos Sistemas de Saúde - OPSS. Meio Caminho Andando. Relatório de Primavera 2018. Lisboa: OPSS;2018. 